\title{
IMPROVED HARDY INEQUALITIES WITH EXACT REMAINDER TERMS
}

\author{
Nguyen Tuan Duy, Nguyen Lam, Nguyen Anh Triet and Weijia Yin
}

Abstract. We set up several identities that imply some versions of the Hardy type inequalities. These equalities give a straightforward understanding of several Hardy type inequalities as well as the nonexistence of nontrivial optimizers. These identities also provide the "virtual" extremizers for many Hardy type inequalities.

Mathematics subject classification (2010): 26D10, 46E35, 35A23.

Keywords and phrases: Hardy type inequalities, Bessel pair, remainder term, optimizer.

\section{REFERENCES}

[1] Adimurthi, N. Chaudhuri And M. Ramaswamy, An improved Hardy-Sobolev inequality and its application, Proc. Amer. Math. Soc. 130 (2002), no. 2, 489-505.

[2] G. Barbatis, S. Filippas And A. Tertikas, A unified approach to improved $L^{p}$ Hardy inequalities with best constants, Trans. Amer. Math. Soc. 356 (2004), no. 6, 2169-2196.

[3] G. Barbatis, S. Filippas and A. Tertikas, Series expansion for $L^{p}$ Hardy inequalities, Indiana Univ. Math. J. 52 (2003), no. 1, 171-190.

[4] K. Bogdan, B. DYDa AND P. KIM, Hardy inequalities and non-explosion results for semigroups, Potential Anal. 44 (2016), 229-247.

[5] H. BRezis AND J. L. VÁZQUeZ, Blow-up solutions of some nonlinear elliptic problems, Rev. Mat. Univ. Complut. Madrid 10, 1997, 443-469.

[6] C. CAZACU AND E. ZuAZUA, Improved multipolar Hardy inequalities, Studies in phase space analysis with applications to PDEs, 35-52, Progr. Nonlinear Differential Equations Appl., 84, Birkhäuser/Springer, New York, 2013.

[7] E. B. Davies, A review of Hardy inequalities, The Maz'ya anniversary collection, Vol. 2 (Rostock, 1998), 55-67, Oper. Theory Adv. Appl., 110, Birkhäuser, Basel, 1999.

[8] J. Dolbeault And B. Volzone, Improved Poincaré inequalities, Nonlinear Anal. 75 (2012), 5985 6001.

[9] N. T. Duy, N. Lam And N. A. Triet, Hardy-Rellich identities with Bessel pairs, Arch. Math. (Basel) 113 (2019), no. 1, 95-112.

[10] N. T. DuY, N. LAm And N. A. TRIET, Hardy and Rellich inequalities with exact missing terms on homogeneous groups, J. Math. Soc. Japan 71 (2019), no. 4, 1243-1256.

[11] N. T. DUY, N. LAM AND N. A. TRIET, Improved Hardy and Hardy-Rellich type inequalities with Bessel pairs via factorizations, J. Spectr. Theory, to appear.

[12] D. E. Edmunds AND H. TRIEBEL, Sharp Sobolev embedding and related Hardy inequalities:the critical case, Math. Nachr. 207 (1999), 79-92.

[13] W. D. Evans AND R. T. LeWIS, Hardy and Rellich inequalities with remainders, J. Math. Inequal. 1, 473-490 (2007).

[14] S. Filippas And A. Tertikas, Optimizing improved Hardy inequalities, J. Funct. Anal. 192 (2002), no. 1, 186-233.

[15] R. FranK AND R. SEIRINGER, Non-linear ground state representations and sharp Hardy inequalities, J. Funct. Anal. 255 (2008), no. 12, 3407-3430.

[16] F. Gazzola, H.-C. Grunau And E. Mitidieri, Hardy inequalities with optimal constants and remainder terms, Trans. Amer. Math. Soc. 356 (2004), no. 6, 2149-2168. 
[17] F. Gesztesy and L. Littlejohn, Factorizations and Hardy-Rellich-type inequalities, Non-linear partial differential equations, mathematical physics, and stochastic analysis, 207-226, EMS Ser. Congr. Rep., Eur. Math. Soc., Zürich, 2018.

[18] N. Ghoussoub And A. Moradifam, Bessel pairs and optimal Hardy and Hardy-Rellich inequalities, Math. Ann. 349 (2011), no. 1, 1-57.

[19] N. GHoussoub AND A. Moradifam, Functional inequalities: new perspectives and new applications, Mathematical Surveys and Monographs, 187. American Mathematical Society, Providence, RI, 2013. xxiv+299.

[20] N. IOKU AND M. Ishiwata, A scale invariant form of a critical Hardy inequality, Int. Math. Res. Not. IMRN 2015, no. 18, 8830-8846.

[21] N. IOKU, M. IshiWATA AND T. OZAWA, Sharp remainder of a critical Hardy inequality, Arch. Math. (Basel) 106 (2016), no. 1, 65-71.

[22] A. Kufner, L. Maligranda And L.-E. Persson, The Hardy Inequality. About its History and Some Related Results, Vydavatelský Servis, Pilsen, 2007.

[23] A. Kufner And L.-E. Persson, Weighted inequalities of Hardy type, World Scientific Publishing Co., Inc., River Edge, NJ, 2003. xviii+357 pp.

[24] N. LAM, A note on Hardy inequalities on homogeneous groups, Potential Anal. 51 (2019), no. 3, 425-435.

[25] N. LAm, Hardy and Hardy-Rellich type inequalities with Bessel pairs, Ann. Acad. Sci. Fenn. Math. 43 (2018), 211-223.

[26] N. LAM, G. LU AND L. ZHANG, Factorizations and Hardy's type identities and inequalities on upper half spaces, Calc. Var. Partial Differential Equations 58 (2019), no. 6, Paper No. 183, 31 pp.

[27] N. LAM, G. LU AND L. ZhANG, Geometric Hardy's inequalities with general distance functions, J. Funct. Anal. 279 (2020), no. 8, 108673, 35 pp..

[28] S. Machihara, T. OZAWA AND H. WADADE, Remarks on the Hardy type inequalities with remainder terms in the framework of equalities, Asymptotic Analysis for Nonlinear Dispersive and Wave Equations, 247-258, Mathematical Society of Japan, Tokyo, Japan, 2019. doi:10.2969/aspm/08110247. https://projecteuclid.org/euclid.aspm/1572545247.

[29] S. Machinara, T. OzaWa And H. WADAde, Scaling invariant Hardy inequalities of multiple logarithmic type on the whole space, J. Inequal. Appl. 2015, 2015:281, 13 pp.

[30] V. H. NGUYEN, New sharp Hardy and Rellich type inequalities on Cartan-Hadamard manifolds and their improvements, Proc. Roy. Soc. Edinburgh Sect. A, 1-30. doi:10.1017/prm.2019.37.

[31] B. OpIC And A. Kufner, Hardy-type inequalities, Pitman Research Notes in Mathematics Series, 219, Longman Scientific \& Technical, Harlow, 1990. xii+333 pp.

[32] M. Ruzhansky And D. Suragan, Anisotropic $L^{2}$-weighted Hardy and $L^{2}$-Caffarelli-KohnNirenberg inequalities, Commun. Contemp. Math. 19 (2017), no. 6, 1750014, 12 pp.

[33] M. RUZHANSKY AND D. SURAGAN, Hardy and Rellich inequalities, identities, and sharp remainders on homogeneous groups, Adv. Math. 317 (2017), 799-822.

[34] M. Ruzhansky and D. Suragan, On horizontal Hardy, Rellich, Caffarelli-Kohn-Nirenberg and p-sub-Laplacian inequalities on stratified groups, J. Differential Equations 262 (2017), no. 3, 17991821.

[35] M. SAno, Scaling invariant Hardy type inequalities with non-standard remainder terms, Math. Inequal. Appl. 21 (2018), no. 1, 77-90.

[36] M. SANO AND F. TAKAHASHI, Scale invariance structures of the critical and the subcritical Hardy inequalities and their improvements, Calc. Var. Partial Differential Equations 56 (2017), no. 3, Art. 69, $14 \mathrm{pp}$.

[37] F. TAKahashi, A simple proof of Hardy's inequality in a limiting case, Arch. Math. (Basel) 104 (2015), no. 1, 77-82. 